\title{
Usefulness of Rehabilitation in Patients with Rotator Cuff Calcific Tendinopathy after Ultrasound-Guided Percutaneous Treatment
}

\author{
Michele Abate Cosima Schiavone Vincenzo Salini \\ Department of Medicine and Science of Aging, University G. d'Annunzio, Chieti-Pescara, Chieti, Italy
}

\section{Key Words}

Rotator cuff calcific tendinopathy - Rehabilitative treatment . Ultrasound

\begin{abstract}
Objective: To evaluate the efficacy of a specific rehabilitation program for patients in whom ultrasound-guided percutaneous treatment (UGPT) was performed for rotator cuff calcific tendinopathy (RCCT). Subjects and Methods: In this prospective observational study, 86 patients ( 22 males and 64 females) with shoulder calcific tendinopathy treated with UGPT were enrolled. At the end of the procedure, a corticosteroid injection into the subacromial-subdeltoid bursa was performed. The patients were then asked to follow a specific rehabilitation protocol (2 times/week for 5 weeks) that focused on mobility, strength and function. At baseline and after 6 weeks, clinical parameters, visual analog scale (VAS) and Constant-Murley scale (CMS) scores and ultrasound (US) features were collected. Results: The mean age of the patients was $48.9 \pm 8.4$ years and their mean BMI was $22.7 \pm 2.1$. Considering the whole cohort, the treatment was effective, with a significant decrease in the VAS score and an improve-
\end{abstract}

ment in the CMS score. Thereafter, on the basis of the compliance to the rehabilitation program (by self-report), 53 and 33 patients were included in the rehabilitation group (Rehab group; performed exercises $\geq 2$ times/week) and the No Rehab group (performed exercises $<2$ times/week), respectively. The comparison between the groups showed that the subjects who performed the exercises regularly had better results in terms of pain and functional recovery, and less associated diseases (e.g. adhesive bursitis and tenosynovitis of the long head of the biceps) than those who were less compliant with the program. Conclusions: UGPT, followed by a specific postprocedure rehabilitation program, was an effective treatment for RCCT.

(c) 2014 S. Karger AG, Basel

\section{Introduction}

Rotator cuff calcific tendinopathy (RCCT) is a common disease characterized by the deposition of carbonate apatite crystals in the rotator cuff tendons $[1,2]$. In the acute phase, it can be highly disabling, with pain that is resistant to conventional therapies (e.g. analgesics, anti-

\begin{tabular}{ll}
\hline KARGER 125/s & $\begin{array}{l}\text { ๑ 2014 S. Karger AG, Basel } \\
1011-7571 / 14 / 0241-0023 \$ 39.50 / 0 \quad \text { Karger }\end{array}$ \\
E-Mail karger@karger.com & $\begin{array}{l}\text { This is an Open Access article licensed under the terms of the } \\
\text { Creative Commons Attribution-NonCommercial 3.0 Un- } \\
\text { www.karger.com/mpp }\end{array}$ \\
& $\begin{array}{l}\text { ported license (CC BY-NC) (www.karger.com/OA-license), } \\
\text { applicable to the online version of the article only. Distribu- } \\
\text { tion permitted for non-commercial purposes only. }\end{array}$
\end{tabular}

Dr. Michele Abate

Department of Medicine and Science of Aging

University G. d'Annunzio, Chieti-Pescara, Via dei Vestini 31

IT-66013 Chieti Scalo (Italy)

E-Mail m.abate@unich.it 
inflammatory drugs and physiotherapy) [3]. Several studies have reported that ultrasound-guided percutaneous treatment (UGPT) is effective for RCCT, with positive results in terms of pain reduction and functional improvement in the short, mid and long term [2, 4-10]. This is an elective, minimally invasive, minimally painful, low-cost technique that requires well-trained hands and does not take long to perform. In nearly all cases, after the aspiration and lavage of the calcific deposits, the injection of steroids into the subacromial-subdeltoid bursa (SSB) is performed to prevent the formation of adhesive bursitis, which can reduce the efficacy of the treatment $[2,4-10]$.

However, less attention has been addressed to the role of a rehabilitation program for the recovery of shoulder function after the UGPT. This procedure is sometimes anecdotally reported $[4,6]$, but its efficacy has not been evaluated. Hence, the aim of this study was to evaluate the efficacy of a specific and progressive exercise protocol on the functional recovery of the shoulders of patients suffering from RCCT who underwent UGPT followed by the injection of steroids into the SSB.

\section{Subjects and Methods}

This was a prospective, observational study performed on 86 consecutive patients (22 males and 64 females) with RCCT treated with UGPT in our echographic unit, followed by a 5-week rehabilitation program, from June 2011 to December 2013. The study was performed according to the Declaration of Helsinki, and informed written consent was obtained from each patient.

To identify rotator cuff calcification, an evaluation was carried out with ultrasound (US) according to a standard protocol utilizing multiplanar scans [11] and by means of a multifrequency (5-14 $\mathrm{MHz}$ ) linear array probe (ProSound Alpha 10, Aloka, Japan). The number, location and size of calcium deposits in each shoulder were recorded. The US appearance of these deposits was classified as hard, soft or fluid, when manifesting with a hyperechoic rim and a strong posterior acoustic shadow, a homogeneous hyperechoic appearance without a posterior acoustic shadow or a thin peripheral hyperechoic rim and a hypoechoic and/or anechoic center, respectively [12]. The involvement of the SSB was identified when accumulation of anechoic fluid, with or without hypoechoic swelling of the synovia and/or bursal wall thickening (adhesive bursitis), appeared within it [13].

The appearance of an anechoic area around the biceps tendon, associated or not with hypoechoic synovial proliferation, was considered as a sign of tenosynovitis [13]. Rotator cuff tears were also assessed by means of a standard protocol and, when present, were classified as partial-thickness tears (i.e. focal hypoechoic discontinuity with irregular margins at the bursal or articular side or located intratendinously) or full-thickness tears (i.e. a full defect in the tendon from the bursal to the articular margin) [11].
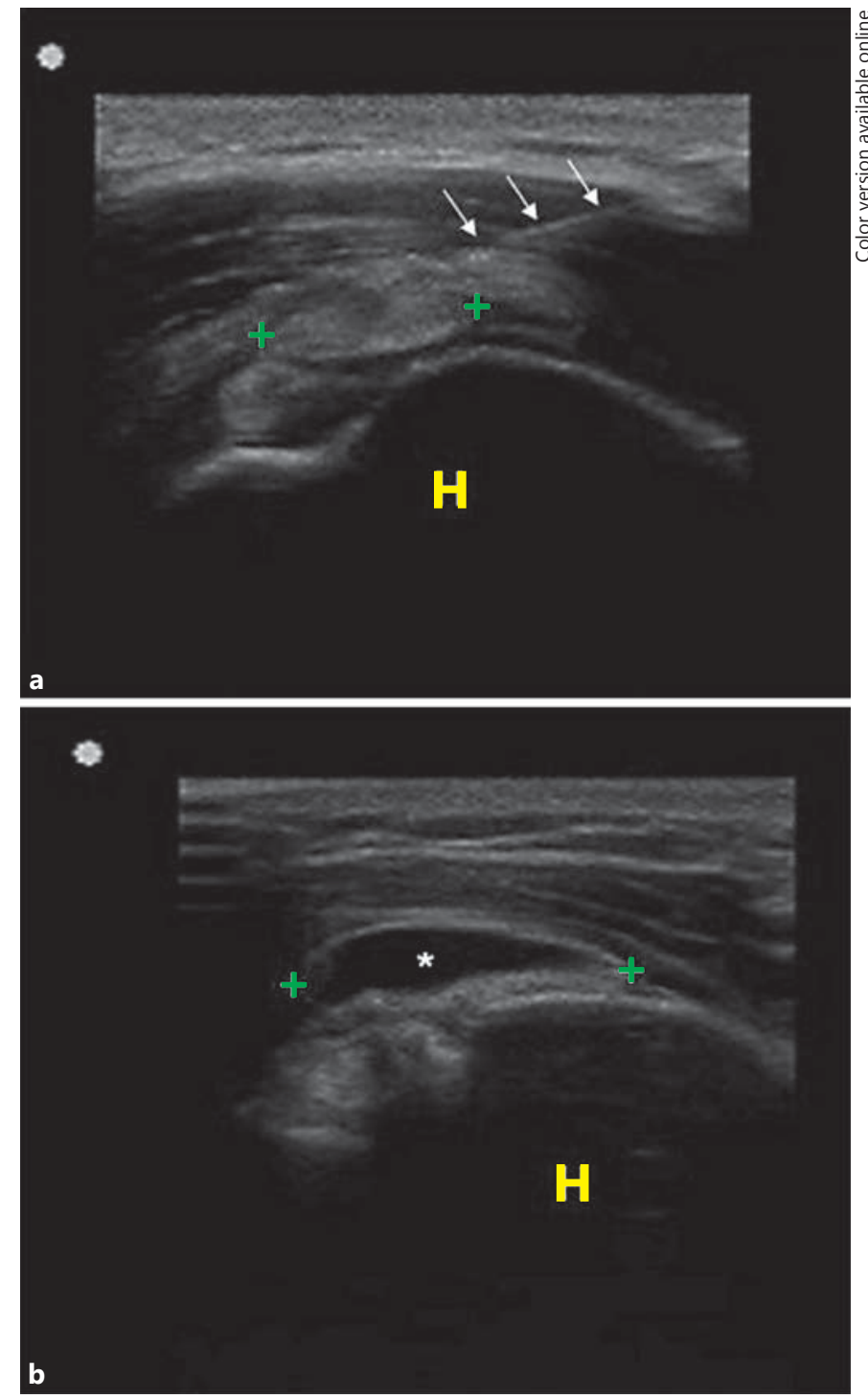

Fig. 1. US appearance of treated calcifications. a A soft calcific deposit $(+)$ appears homogeneous and hyperechoic without posterior acoustic shadow. The needle position was also observed (arrows). b A fluid calcification characterized by a thin peripheral hyperechoic rim $(+)$ and an anechoic center $\left(^{*}\right)$ is observed. $\mathrm{H}=$ Humeral head.

Pain during the previous week was registered on a visual analog scale (VAS; $0=$ no pain and $10=$ unbearable pain). Inclusion criteria were patients in their algic or hyperalgic phase (pain score $\geq 3$ ) with the US appearance of soft and/or fluid calcific deposits (>5 mm; fig. 1). Patients were excluded if they had an ipsilateral concomitant cuff tear or had received previous extracorporeal shock wave treatment, physical therapy or local injection of steroids.

Before treatment, demographic and anthropometric data (sex, age and BMI) and symptom duration were collected. The Con- 
stant-Murley scale (CMS), based on the evaluation of two subjective (pain and activities of daily living) and two objective [joint range of motion (ROM) and strength] items, was used to evaluate shoulder function during the preceding week [14].

US examinations and UGPTs were performed by the same operator (M.A., with 6 years of experience in musculoskeletal US and US-guided musculoskeletal interventional procedures). The UGPT was performed with the patient lying on a bed on his/her side, with the shoulder to be treated upwards. Under sterile conditions, $10 \mathrm{ml}$ of $2 \%$ mepivacaine chloridrate were injected into the subcutaneous tissues, into the SSB and then around the calcification. Afterwards, two 18-gauge needles were inserted inside the calcific deposit $[9,10]$. Once their correct placement had been verified, one of them was connected with a $20-\mathrm{ml}$ syringe (Luer lock) through a 3 -way valve; the calcification was then washed with $0.9 \%$ saline solution, which allowed the breaking up, dissolution and elimination of calcium through the other needle (fig. 2). The lavage was repeated several times until the flushed fluid was completely free of visible calcium $[9,10]$. When the treatment was complete, the tip of one of the two needles was placed into the SSB, and $1 \mathrm{ml}$ of methylprednisolone acetate $(40 \mathrm{mg} / \mathrm{ml})$ mixed with $3 \mathrm{ml}$ of $2 \%$ mepivacaine chloridrate was injected. Finally, a medicated plaster was applied.

After the treatment, each patient was kept under observation for approximately $30 \mathrm{~min}$ and then discharged from the unit. $\mathrm{Pa}$ tients were instructed to apply ice packs for up to 2 days after the procedure once at home, and the use of antibiotics (amoxicillin and clavulanic acid: $875+125 \mathrm{mg}, 2$ tablets/day) for 5 days was prescribed. Pain-killers, when pain was intolerable, were allowed. Thereafter, the patients were instructed to follow a specific and progressive rehabilitation protocol (explicit instructions consisting of several phases were provided; fig. 3). In the first phase (week $1)$, stretching, pendulum and isometric exercises and self-assisted exercises in the pain-free ROM (i.e. anterior elevation, internal/ external rotation, abduction and retroposition) were started. In the second week, active exercises to increase the ROM (especially in internal rotation and abduction) and stretching of the anterior and posterior scapulohumeral capsule were introduced. The kinematics of the scapulothoracic joint were also addressed. In the third phase (week 3), strengthening and endurance exercises, using both elastic bands and weights, were started. Strength, resistance and power were gradually increased in the next phase (weeks $4 / 5$ ). In patients doing sport, isokinetic exercises in internal/external rotation in a neutral position and in abduction/adduction as well as sport-specific movements were allowed. Patients were asked to perform the program with the aid of a physiotherapist 2 times/ week (for about $1 \mathrm{~h}$ ) for 5 weeks. They were also encouraged to continue with the prescribed exercises at home as much as possible.

After 6 weeks, clinical (VAS and CMS) and US assessments were repeated, and patients' satisfaction was registered by means of a 5-point Likert scale: not satisfied at all, slightly satisfied, somewhat satisfied, very satisfied and extremely satisfied [15].

On the basis of compliance to the rehabilitation program by self-report, patients were divided into 2 groups: the Rehab group (performed exercises $\geq 2$ times/week) and the No Rehab group (performed exercises $<2$ times/week). The demographic, US and clinical data before and after 6 weeks were compared. Data are reported as mean \pm SD for continuous variables, whereas categorical and dichotomous variables are reported as frequencies and per-
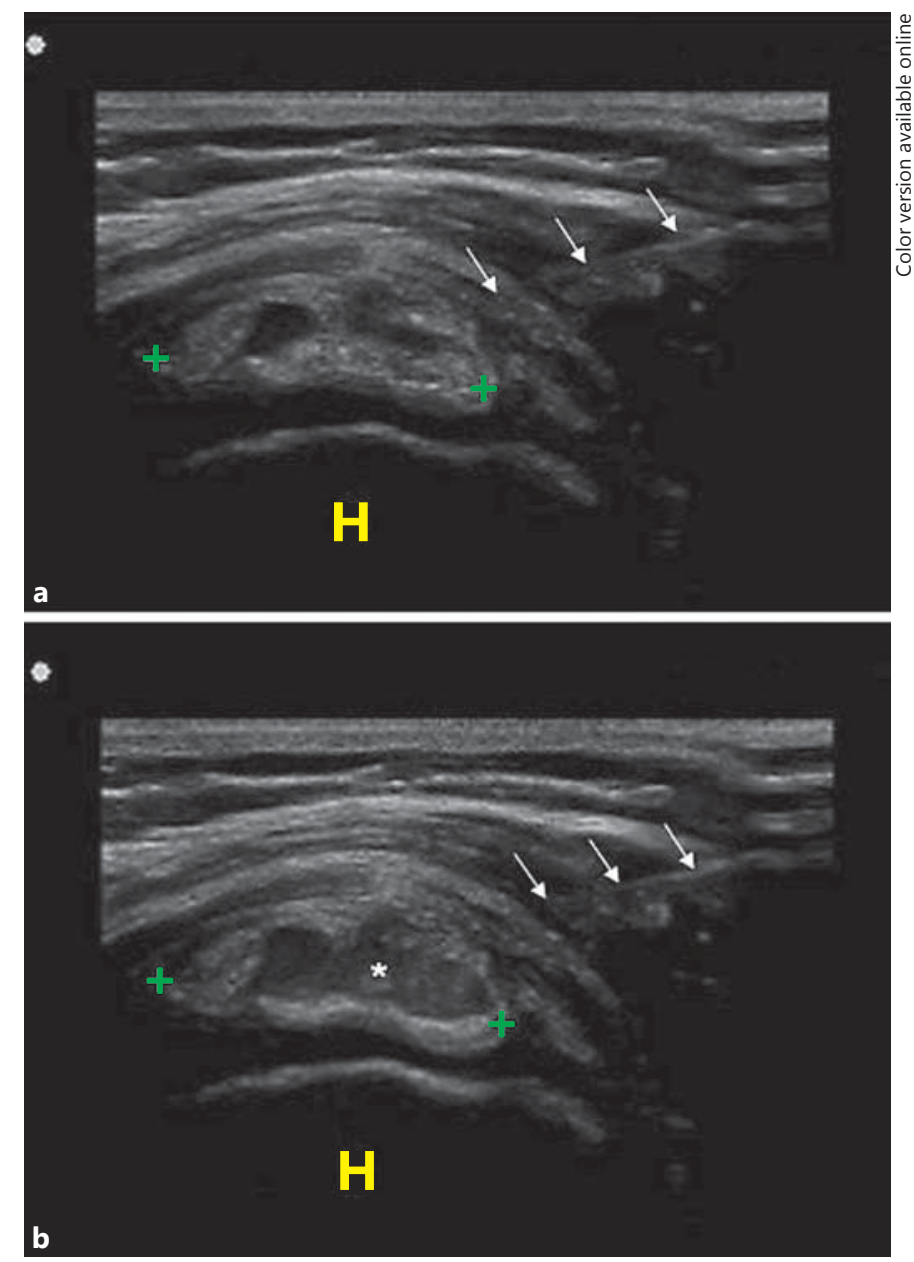

Fig. 2. US-guided lavage of calcifications. a After the placement of the needles (arrows) inside the calcification $(+)$, the calcific deposit is washed with saline solution. $\mathbf{b}$ This creates a swirl $\left(^{*}\right.$, nebular area) inside the calcification and allows the breaking up, dissolution and elimination of calcium through the other needle. $\mathrm{H}=$ Humeral head.

centages. The 2-sample Student t test was used to compare continuous variables when the distribution of data was normal, and otherwise the Wilcoxon rank sum test was used. The $\chi^{2}$ test was used to evaluate associations between categorical data. The significance level was determined at $\mathrm{p}<0.05$.

\section{Results}

The patients' demographic and clinical data and the US characteristics of the calcifications are reported in table 1 . The procedures were well tolerated and no complications were observed during or after treatment. Taking 
Week 1

- Pendulum exercises

- Active assisted exercises (anterior elevation, external rotation and retroposition)

- Isometric endurance exercises (external/internal rotation and abduction, elbow flexion)

- Stretching exercises [passive mobilization (flexion, abduction, external/internal rotation)]

Week 2

- Continue with first week exercises while improving ROM

- Stretching of the anterior and posterior shoulder capsule

Week 3

- Continue with exercises from weeks 1 and 2

- Strengthening exercises [with elastic bands and weights (anterior elevation,

external/internal rotation, retroposition and push-up)]

- Start with opposition exercises by means of upper limb ergometer

Week 4

- Continue with strengthening exercises while increasing strength, endurance and power

- Isokinetic exercises (external/internal rotation and abduction/adduction)

Week 5

- Sport-specific exercises

Fig. 3. Rehabilitative program after UGPT.

Table 1. Demographic and clinical characteristics of all patients at baseline

\begin{tabular}{lc}
\hline Patients & 86 \\
Age, years & $48.9 \pm 8.4$ \\
Sex & $22(25.5)$ \\
$\quad$ Men & $64(74.4)$ \\
$\quad$ Women & $22.7 \pm 2.1$ \\
BMI & $4.6 \pm 2.8$ \\
Symptom duration, months & \\
Affected side & $47(54.6)$ \\
$\quad$ Right & $39(45.3)$ \\
$\quad$ Left & $64(74.4)$ \\
Tendon involved & $19(22)$ \\
$\quad$ Supraspinatus & $3(3.4)$ \\
Infraspinatus & $14.7 \pm 4.4$ \\
$\quad$ Subscapolaris & \\
Dimension, mm & $70(81.3)$ \\
Calcification appearance & $16(18.6)$ \\
$\quad$ Soft & \\
$\quad$ Fluid & $78(90.6)$ \\
Associated diseases & $6.7 \pm 1.1$ \\
$\quad$ Bursitis & $38.6 \pm 15$ \\
VAS score &
\end{tabular}

Figures denote $\mathrm{n}(\%)$ or means \pm SD. into account the whole group, a significant decrease in the VAS score $(6.7 \pm 1.1$ vs. $2.6 \pm 1.2, \mathrm{p}<0.0000)$ and an improvement in the CMS score $(38.6 \pm 15$ vs. $79.5 \pm 6.2, \mathrm{p}<$ $0.0000)$ were observed.

At the follow-up (after 6 weeks), 53 patients reported (by self-report) to have regularly performed the rehabilitation program (Rehab group), while the remaining 33 participants reported that they had not (No Rehab). No differences in the baseline values were observed between the 2 groups, with the exception of a small prevalence of the right affected side in the No Rehab group (table 2). When comparing the 2 groups, a greater, statistically significant improvement in the VAS and CMS scores was observed in the Rehab group, while associated diseases (subacromial-subdeltoid adhesive bursitis, tenosynovitis of the long head of the biceps, or both) were more frequently found in the No Rehab group (fig. 4; table 2). Finally, patients in the Rehab group were more satisfied (i.e. extremely and very satisfied) with the treatment (77.3 vs. $33.3 \%$ in the No Rehab group, $\mathrm{p}<0.0000$ ). A further analysis comparing patients with soft and fluid calcifications did not show any difference in the functional outcomes (CMS and VAS scores) at the final follow-up (data not reported). 
Table 2. Differences between patients who followed the rehabilitation protocol properly and those who did not

\begin{tabular}{|c|c|c|c|}
\hline & Rehab & No Rehab & $\mathrm{p}$ \\
\hline Patients & 53 & 33 & \\
\hline Age, years & $48.6 \pm 8.8$ & $49.4 \pm 7.8$ & 0.3 \\
\hline \multicolumn{4}{|l|}{ Sex } \\
\hline Men & $13(24.5)$ & $9(27.2)$ & 0.7 \\
\hline Women & $40(75.4)$ & $24(72.7)$ & \\
\hline BMI & $22.8 \pm 2.3$ & $22.5 \pm 1.7$ & 0.2 \\
\hline Symptom duration, months & $4.5 \pm 2.9$ & $4.9 \pm 2.8$ & 0.2 \\
\hline \multicolumn{4}{|l|}{ Affected side } \\
\hline Right & $24(45.2)$ & $23(69.6)$ & 0.02 \\
\hline Left & $29(54.7)$ & $10(30.3)$ & \\
\hline \multicolumn{4}{|l|}{ Tendon involved } \\
\hline Supraspinatus & $38(71.6)$ & $26(78.7)$ & 0.4 \\
\hline Infraspinatus & $13(24.5)$ & $6(18.1)$ & 0.4 \\
\hline Subscapolaris & $2(3.7)$ & $1(3)$ & 0.8 \\
\hline Dimension, mm & $14.7 \pm 4.3$ & $14.8 \pm 4.5$ & 0.4 \\
\hline \multicolumn{4}{|l|}{ Calcification appearance } \\
\hline Soft & $44(83)$ & $26(78.7)$ & 0.6 \\
\hline Fluid & $9(16.9)$ & $7(21.2)$ & 0.6 \\
\hline Associated diseases & $5(9.4)$ & $10(30.3)$ & 0.01 \\
\hline Bursitis & $3(5.6)$ & $6(18.1)$ & \\
\hline Tenosynovitis & $1(1.8)$ & $2(6)$ & \\
\hline Both & $1(1.8)$ & $2(6)$ & \\
\hline \multicolumn{4}{|l|}{ VAS score } \\
\hline Pretreatment & $6.8 \pm 1$ & $6.5 \pm 1.2$ & 0.1 \\
\hline Posttreatment & $2.1 \pm 1.1$ & $3.3 \pm 0.9$ & 0.0000 \\
\hline \multicolumn{4}{|l|}{ CMS score } \\
\hline Pretreatment & $39 \pm 15.1$ & $38 \pm 15.1$ & 0.3 \\
\hline Posttreatment & $82.4 \pm 5$ & $74.8 \pm 4.9$ & 0.0000 \\
\hline \multicolumn{4}{|l|}{ Satisfaction } \\
\hline Extremely satisfied & $26(49)$ & $4(12.1)$ & 0.000 \\
\hline Very satisfied & $15(28.3)$ & $7(21.2)$ & 0.46 \\
\hline Somewhat satisfied & $5(9.4)$ & $10(30.3)$ & 0.01 \\
\hline Slightly satisfied & $7(13.2)$ & $12(22.6)$ & 0.01 \\
\hline Not at all satisfied & - & - & - \\
\hline
\end{tabular}

Figures denote $\mathrm{n}(\%)$ or means $\pm \mathrm{SD}$.
UGPT and then rehabilitative treatment were performed. However, it is noteworthy to point out that in their study, they included other groups for comparison and the patients performed the exercises at home after having been instructed by a therapist [4].

The better performance of the Rehab group was due to the positive effects of the exercises, as reported previously $[16,17]$. Indeed, throughout the period of the program, the patients were able to almost completely recover the mobility of the shoulder (with ROM exercises) [17-19] and reduce the symptoms associated with the impingement syndrome (with pendulum exercises and strengthening of the humeral depressor muscles) [20]. Moreover, the specific exercises addressing the glenohumeral and scapulothoracic joints (stretching and tasks) also improved the kinematic and neuromuscular control of the shoulder with the recovery of a more physiological movement $[20,21]$. The strengthening and endurance exercises of the rotator cuff muscles, performed during the final phases of the program, were effective in stabilizing the humeral head on the glenoid and in the recovery from disuse atrophy and deconditioning usually associated with chronic shoulder pain [22]. Finally, exercise was also effective in mitigating the pain associated with the muscular spasms frequently observed in this disease.

Besides the positive effects of the exercise, the better outcomes could also be due to the positive effect of rehabilitation in lowering the incidence of associated complications. Indeed, in the Rehab group, a lower incidence of inflammatory pathologies, such as subacromial-subdeltoid adhesive bursitis, tenosynovitis of the long head of the biceps, or both, were observed at the US examination after 6 weeks. These pathologies can be ascribed to the impingement syndrome that produces the compression and irritation of the soft tissues (mainly the SSB) during elevation of the arm [23] and to the overload of the long head of the biceps [24]. Other possible explanations could be poor stretching or conditioning before exercise, and the strength and flexibility imbalance of the scapula muscles which may lead to impingement of the SSB that then becomes inflamed, swollen and thick [25]. Several authors have shown that adhesive bursitis and/or tenosynovitis are correlated with a lesser efficacy of the UGPT [4, $9,10]$.

This study has several limitations. Firstly, a control group with no rehabilitation at all was lacking, but this was a deliberate decision because we considered it unethical to exclude a priori some patients from the rehabilitation program. Secondly, patients were divided into 

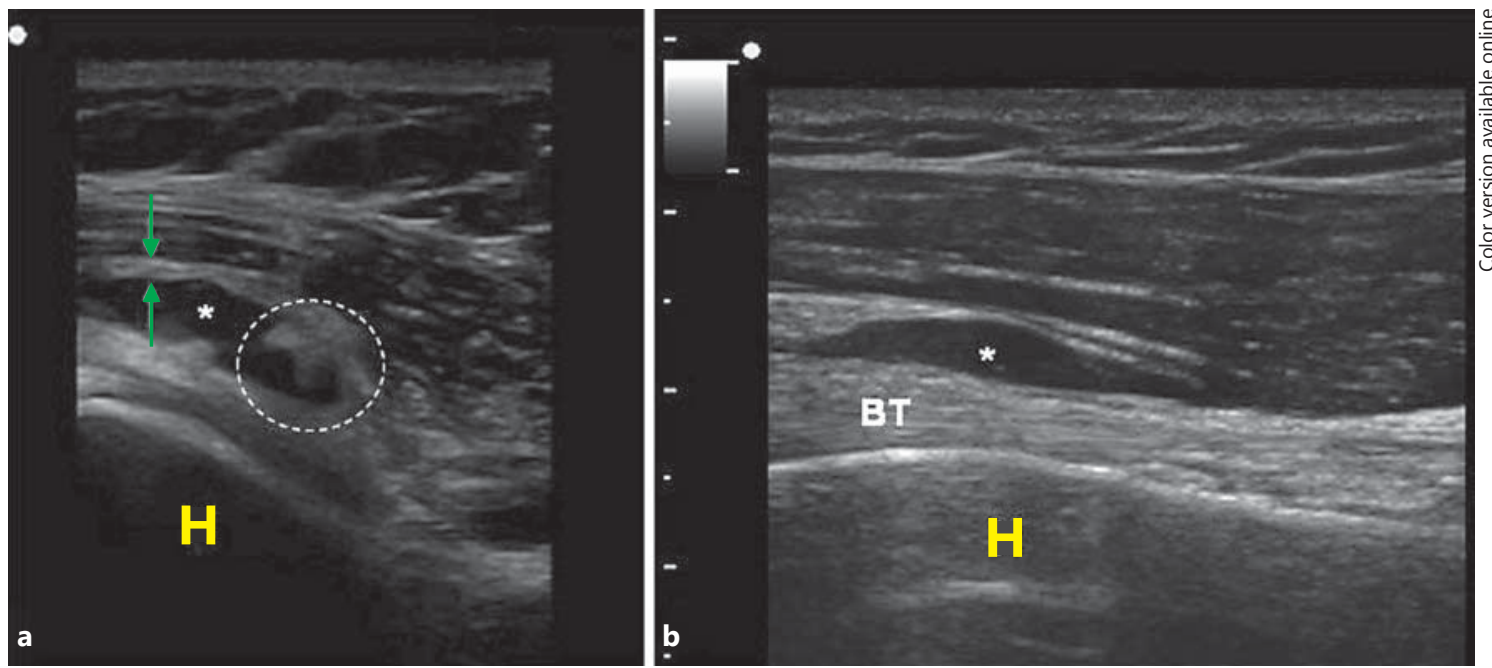

Fig. 4. US appearance of associated diseases. a The presence of an anechoic effusion (*) inside the SSB, associated with bursal wall thickening (arrows) and synovial hyperplasia, is an expression of adhesive bursitis. $\mathbf{b}$ Tenosynovitis of the long head of the biceps is characterized by fluid effusion $\left(^{*}\right)$ above the biceps tendon which expands the synovial sheet. BT = Biceps tendon; $\mathrm{H}=$ humeral head.

2 groups on the basis of their compliance to the rehabilitation program only by self-reporting, because the treatment was performed outside our US unit. Thirdly, the relatively short duration of the follow-up did not allow an evaluation of how long the therapeutic effect was maintained. The strength of our study was the large number of patients treated and evaluated with standard procedures.

\section{Conclusion}

The UGPT followed by a specific postprocedure rehabilitation program was an effective treatment for RCCT.

\section{Disclosure Statement}

The authors declare that there are no conflicts of interest.

\section{References}

$\checkmark 1$ Hamada J, Ono W, Tamai K, et al: Analysis of calcium deposits in calcific periarthritis. J Rheumatol 2001;28:809-813.

-2 del Cura JL, Torre I, Zabala R, et al: Sonographically guided percutaneous needle lavage in calcific tendinitis of the shoulder: short- and long-term results. Am J Roentgenol 2007;189:W128-W134.

3 Hurt G, Baker CL Jr: Calcific tendinitis of the shoulder. Orthop Clin North Am 2003;34: 567-575.

-4 Fusaro I, Orsini S, Diani S, et al: Functional results in calcific tendinitis of the shoulder treated with rehabilitation after ultrasonicguided approach. Musculoskelet Surg 2011; 95:S31-S36.

5 Lin JT, Adler RS, Bracilovic A, et al: Clinical outcomes of ultrasound-guided aspiration and lavage in calcific tendinosis of the shoulder. HSS J 2007;3:99-105.
Galletti S, Magnani M, Rotini R, et al: The echo-guided treatment of calcific tendinitis of the shoulder. Chir Organi Mov 2004;89:319323.

7 Farin PU, Jaroma H, Soimakallio S: Rotator cuff calcifications: treatment with US-guided technique. Radiology 1995;195:841-843.

$>8$ De Zordo T, Ahmad N, Ødegaard F, et al: USguided therapy of calcific tendinopathy: clinical and radiological outcome assessment in shoulder and non-shoulder tendons. Ultraschall Med 2011;32:S117-S123.

-9 Serafini G, Sconfienza LM, Lacelli F, et al: Rotator cuff calcific tendonitis: short-term and 10 -year outcomes after two-needle US-guided percutaneous treatment - nonrandomized controlled trial. Radiology 2009;252:157-164.
10 Sconfienza LM, Bandirali M, Serafini G, et al: Rotator cuff calcific tendinitis: does warm saline solution improve the short-term outcome of double-needle US-guided treatment? Radiology 2012;262:560-566.

11 Abate M, Schiavone C, Di Carlo L, et al: Prevalence of and risk factors for asymptomatic rotator cuff tears in postmenopausal women. Menopause 2014;21:275-280.

12 Farin PU, Jaroma H: Sonographic findings of rotator cuff calcifications. J Ultrasound Med 1995;14:7-14.

13 Abate M, Schiavone C, Salini V: Sonographic evaluation of the shoulder in asymptomatic elderly subjects with diabetes. BMC Musculoskelet Disord 2010;11:278.

14 Constant CR, Murley AH: A clinical method of functional assessment of the shoulder. Clin Orthop Relat Res 1987;214:160-164. 
15 Kolarz G1, Kotz R, Hochmayer I: Long-term benefits and repeated treatment cycles of intra-articular sodium hyaluronate (Hyalgan) in patients with osteoarthritis of the knee. Semin Arthritis Rheum 2003;32:310-319.

16 Ludewig PM, Borstad JD: Effects of a home exercise programme on shoulder pain and functional status in construction workers. Occup Environ Med 2003;60:841-849.

17 Park KN, Kwon OY, Weon JH, et al: Comparison of the effects of local cryotherapy and passive cross-body stretch on extensibility in subjects with posterior shoulder tightness. J Sports Sci Med 2014;13:84-90.
18 Park SI, Choi YK, Lee JH, et al: Effects of shoulder stabilization exercise on pain and functional recovery of shoulder impingement syndrome patients. J Phys Ther Sci 2013;25: 1359-1362.

19 Mahomed S1, Al-Obaidi S, Al-Zoabi B: Outcome measures and psychomotor skills related to shoulder conditions for clinical orthopedic training. Med Princ Pract 2008;17:481485.

20 Marzetti E, Rabini A, Piccinini G, et al: Neurocognitive therapeutic exercise improves pain and function in patients with shoulder impingement syndrome: a single-blind randomized controlled clinical trial. Eur J Phys Rehabil Med 2014;50:255-264.

21 Suprak DN, Bohannon J, Morales G, et al: Scapular kinematics and shoulder elevation in a traditional push-up. J Athl Train 2013;48: 826-835.
22 Warby SA, Pizzari T, Ford JJ, et al: The effect of exercise-based management for multidirectional instability of the glenohumeral joint: a systematic review. J Shoulder Elbow Surg 2014;23:128-142.

23 Umer M, Qadir I, Azam M: Subacromial impingement syndrome. Orthop Rev (Pavia) 2012;4:e18.

24 Stevens K, Kwak A, Poplawski S: The biceps muscle from shoulder to elbow. Semin Musculoskelet Radiol 2012;16:296-315.

25 Tagg CE, Campbell AS, McNally EG: Shoulder impingement. Semin Musculoskelet Radiol 2013;17:3-11. 